\title{
DUALITY ON NONCOMPACT MANIFOLDS AND COMPLEMENTS OF TOPOLOGICAL KNOTS
}

\author{
GERARD A. VENEMA
}

(Communicated by James E. West)

\begin{abstract}
Let $\Sigma$ be the image of a topological embedding of $S^{n-2}$ into $S^{n}$. In this paper the homotopy groups of the complement $S^{n}-\Sigma$ are studied. In contrast with the situation in the smooth and piecewise-linear categories, it is shown that the first nonstandard homotopy group of the complement of such a topological knot can occur in any dimension in the range 1 through $n-2$. If the first nonstandard homotopy group of the complement occurs above the middle dimension, then the end of $S^{n}-\Sigma$ must have a nontrivial homotopy group in the dual dimension. The complement has the proper homotopy type of $S^{1} \times \mathbb{R}^{n-1}$ if both the complement and the end of the complement have standard homotopy groups in every dimension below the middle dimension.

A new form of duality for noncompact manifolds is developed. The duality theorem is the main technical tool used in the paper.
\end{abstract}

\section{INTRODUCTION}

Let $h: S^{n-2} \rightarrow S^{n}$ be a topological embedding. We will denote the image set $h\left(S^{n-2}\right)$ by $\Sigma$ and refer to $\Sigma$ as a topological knot. In this paper we will study the homotopy groups of the knot complement $S^{n}-\Sigma$. The standard unknotted $(n-2)$-sphere $S^{n-2} \subset S^{n}$ satisfies $\pi_{i}\left(S^{n}-S^{n-2}\right) \cong \pi_{i}\left(S^{1}\right)$ for every $i$, so we refer to $\pi_{i}\left(S^{n}-\Sigma\right)$ as standard if $\pi_{i}\left(S^{n}-\Sigma\right) \cong \pi_{i}\left(S^{1}\right)$. We will answer two questions about homotopy groups of complements of topological knots. First, in which dimension could the first nonstandard homotopy group of the complement appear? Second, under what conditions will all the homotopy groups of the complement be standard?

In case $h$ is a smooth embedding, the situation is well understood. For each $k, 1 \leq k<\frac{n}{2}$, there is a smooth embedding $h: S^{n-2} \rightarrow S^{n}$ such that $\pi_{i}\left(S^{n}-\Sigma\right) \cong \pi_{i}\left(S^{1}\right)$ for $1 \leq i<k$ but $\pi_{k}\left(S^{n}-\Sigma\right) \neq \pi_{k}\left(S^{1}\right)$; see [13, Theorem 2], for example. On the other hand, if $h$ is smooth and $\pi_{i}\left(S^{n}-\Sigma\right) \cong \pi_{i}\left(S^{1}\right)$ for $1 \leq i<\frac{n}{2}$, then $S^{n}-\Sigma$ has the homotopy type of $S^{1}$ ([10] or [7, p. 74]). This last statement, sometimes referred to as Levine's Theorem, is proved by showing that the homology of the universal cover of $S^{n}-\Sigma$ vanishes. If $n$ is odd, this follows quite easily from Poincaré Duality. If $n$ is even, more care

Received by the editors March 22, 1994.

1991 Mathematics Subject Classification. Primary 57M30, 55Q05, 55M05.

Key words and phrases. Knot complement, homotopy groups, duality. 
is needed to prove that the homology group in the middle dimension vanishes. One approach is to use "Milnor Duality" [12]. Milnor shows that, under certain conditions, an infinite cyclic cover of a compact $n$-manifold will have some of the duality properties of an $(n-1)$-manifold. His theorem applies in this case to show that the homology in dimension $\frac{n}{2}$ is isomorphic to the cohomology in dimension $\left(\frac{n}{2}-1\right)$ and is, therefore, trivial.

In this paper we study similar questions for topological embeddings. Our first result shows that, for topological embeddings, the first nonstandard homotopy group can appear above the middle dimension. In fact, the first nonstandard group can appear in any dimension below $n-1$.

Example 1.1. For each $n$ and $k$ with $1 \leq k \leq n-2$ there exists a topological embedding $h: S^{n-2} \rightarrow S^{n}$ such that $\pi_{i}\left(S^{n}-h\left(S^{n-2}\right)\right) \cong \pi_{i}\left(S^{1}\right)$ for $i<k$ but $\pi_{k}\left(S^{n}-h\left(S^{n-2}\right)\right) \neq \pi_{k}\left(S^{1}\right)$. The embedding is smooth except at one point.

Example 1.1 is a generalization of [9, Example 1.1]. If $k \geq \frac{n}{2}$, the embedding in Example 1.1 above must necessarily be wild (because of Levine's Theorem, above). This wildness is manifest in the homotopy groups of the end of $S^{n}-\Sigma$. (See $\S 3$ for the definition of an end of a manifold and $\S 4$ for the definition of homotopy groups of the end.) Our next result shows that there is a definite relationship between the homology groups of the universal cover of $S^{n}-\Sigma$ and the homotopy groups of the end of $S^{n}-\Sigma$.

Theorem 1.2. Suppose $h: S^{n-2} \rightarrow S^{n}$ is a topological embedding. Let $\Sigma$ denote $h\left(S^{n-2}\right)$, let $W=S^{n}-\Sigma$, let $\widetilde{W}$ denote the infinite cyclic cover of $W$, and let $\epsilon$ denote the end of $W$. If there exists an integer $k \geq 2$ such that $\pi_{i}(\epsilon) \cong \pi_{i}\left(S^{1}\right)$ for $i \leq n-k-1$, then $H_{k}(\widetilde{W} ; F) \cong \widetilde{H}^{n-k-1}(\widetilde{W} ; F)$ for every field $F$.

We can therefore control all the homotopy groups of $W$ by controlling those in low dimensions of both $W$ and $\epsilon$.

Corollary 1.3. Suppose $h: S^{n-2} \rightarrow S^{n}$ is a topological embedding. Let $\Sigma$ denote $h\left(S^{n-2}\right)$, let $W=S^{n}-\Sigma$, let $\widetilde{W}$ denote the infinite cyclic cover of $W$, and let $\epsilon$ denote the end of $W$. If there exists an integer $k \geq 0$ such that $\pi_{i}(W) \cong$ $\pi_{i}(\epsilon) \cong \pi_{i}\left(S^{1}\right)$ for $i \leq k$, then $H_{i}(\widetilde{W} ; \mathbb{Z})=0$ for $i \geq n-k-1$.

Corollary 1.4. Suppose $h: S^{n-2} \rightarrow S^{n}$ is a topological embedding. Let $\Sigma$ denote $h\left(S^{n-2}\right)$, let $W=S^{n}-\Sigma$, and let $\epsilon$ denote the end of $W$. If $\pi_{i}(W) \cong \pi_{i}(\epsilon) \cong$ $\pi_{i}\left(S^{1}\right)$ for $i<\frac{n}{2}$, then $W$ has the homotopy type of $S^{1}$.

Conversely, the next theorem asserts that we can control the homotopy groups of the end of $W$ by controlling the global homotopy groups of $W$. In particular, if $\pi_{i}(W) \cong \pi_{i}\left(S^{1}\right)$ for every $i$, then the first nonstandard homotopy group of the end must appear in dimension 1 (or not at all). This is surprising in view of Example 1.1 which says that the first nonstandard homotopy group of the entire complement can appear in any dimension; one might expect that the first nonstandard homotopy group of the end of the complement could also appear in any dimension. A more geometric version of the following result was first discovered by Hollingsworth and Rushing [5] in dimensions $n \geq 5$. The 4-dimensional case is covered in [8] and [9]. We include a proof of the general case here in order to demonstrate that the theorem is a consequence of rather elementary duality arguments and does not require the geometric machinery of [5]. 
Theorem 1.5. Suppose $h: S^{n-2} \rightarrow S^{n}$ is a topological embedding. Let $\Sigma$ denote $h\left(S^{n-2}\right)$, let $W=S^{n}-\Sigma$, and let $\epsilon$ denote the end of $W$. If $\pi_{i}(W) \cong \pi_{i}\left(S^{1}\right)$ for every $i$ and $\pi_{1}(\epsilon) \cong \mathbb{Z}$, then $\pi_{i}(\epsilon)=0$ for $1<i<n-2$ and $\pi_{n-2}(\epsilon) \cong \mathbb{Z}$.

When we combine Corollary 1.4 with Theorem 1.5 we get a theorem for topological knots that is analogous to Levine's Theorem for smooth knots.

Corollary 1.6. Suppose $h: S^{n-2} \rightarrow S^{n}$ is a topological embedding. Let $\Sigma$ denote $h\left(S^{n-2}\right)$, let $W=S^{n}-\Sigma$, and let $\epsilon$ denote the end of $W$. If $\pi_{i}(W) \cong \pi_{i}(\epsilon) \cong$ $\pi_{i}\left(S^{1}\right)$ for $i<\frac{n}{2}$, then $W$ has the proper homotopy type of $S^{1} \times \mathbb{R}^{n-1}$.

It should be noted that it is possible for a topological knot complement to have the homotopy type of $S^{1}$ even though the fundamental group at the end is bad; see $[4, \S 5]$ for examples. It is also possible to construct examples that have the fundamental group at the end infinite cyclic but higher homotopy groups at the end nontrivial (just take a connected sum of a null sequence of smooth examples such as those in [13, Theorem 2]), but such examples must necessarily have nonstandard global homotopy groups as well.

It is relatively easy to prove a weak version of Corollary 1.3 using ordinary Poincaré Duality. But this version would have a weaker conclusion: starting with the hypotheses stated in the theorem, we would only be able to conclude that $H_{i}(\widetilde{W})=0$ for $i \geq n-k$. In order to win the extra dimension we need a noncompact version of Milnor Duality. Milnor's proof [12] uses very strongly the fact that he is working in a cover of a compact polyhedral manifold and hence really only applies in case the knot is smooth or piecewise linear. We will prove an analogous theorem that applies to certain noncompact manifolds. Our version is customized to the setting in which we wish to apply it. Other noncompact variations on Milnor Duality are possible; these could be analogous to some of the compact variations described in [6, Theorem 2.3] and would depend on the coefficients used. Before stating the theorem we must introduce some notation and terminology.

Assume $W$ is a noncompact piecewise-linear $n$-manifold with one end $\epsilon$ (again, see $\S 3$ for a definition of end), and let $p: \widetilde{W} \rightarrow W$ denote an infinite cyclic cover. If $U$ is any subset of $W$, we use $U^{*}$ to denote $p^{-1}(U) \subset \widetilde{W}$. As in Milnor's original work, it is convenient to use coefficients in a field $F$. We define special cohomology groups of $\widetilde{W}$ associated with the end $\epsilon$.

Definition. We define $H^{k}(\widetilde{W}, \tilde{\epsilon} ; F)=\lim H^{k}\left(\widetilde{W}, U^{*} ; F\right)$, where the limit is taken over all neighborhoods $U$ of the end $\epsilon$.

In the case of homology, we do not wish to pass to the limit. Instead we use $H_{k}(\widetilde{W}, \tilde{\epsilon} ; F)$ to denote the the inverse system $\left\{H_{k}\left(\widetilde{W}, U^{*} ; F\right)\right\}$, where $U$ is a neighborhood of $\epsilon$ and the system is partially ordered by inclusion.

Definition. We will say that $H_{k}(\widetilde{W}, \tilde{\epsilon} ; F)$ is profinitely generated over $F$ if for every neighborhood $U$ of $\epsilon$ there exists a neighborhood $V$ of $\epsilon, V \subset U$, such that the image of $H_{k}\left(\widetilde{W}, V^{*} ; F\right)$ in $H_{k}\left(\widetilde{W}, U^{*} ; F\right)$ is finitely generated over $F$.

The following theorem is our noncompact version of Milnor's duality theorem. It is the main technical tool used in the proof of Theorem 1.2, but it applies to spaces other than knot complements as well. 
Theorem 1.7. Suppose that $W$ is a connected $P L$ n-manifold with one end $\epsilon$ and that $\widetilde{W}$ is an infinite cyclic cover of $W$. If $H_{i}(\widetilde{W}, \tilde{\epsilon} ; F)$ is profinitely generated over $F$ for $n-k-2 \leq i \leq n-k$, then $H_{k}(\widetilde{W} ; F) \cong H^{n-k-1}(\widetilde{W}, \tilde{\epsilon} ; F)$.

\section{Construction OF THE WILD KNOTS}

In this section we construct topological knots with the properties specified in Example 1.1. The case $k=1$ of Example 1.1 is adequately covered by the smooth examples, so we will assume for the remainder of this section that $k$ is a fixed integer in the range $2 \leq k \leq n-2$. The construction is a generalization of that in [9] and consists of two parts. First, we construct an open subset $W$ of $S^{n}$ that has the unusual homotopy properties necessary to be the complement of the knot we seek. Second, we explain how to re-embed $W$ in $S^{n}$ so that $S^{n}-W$ is a topological $(n-2)$-sphere.

We begin by constructing the knot complement $W$. Since $k \geq 2$, we know that we must have $\pi_{1}(W)$ infinite cyclic. In addition, $W$ must have $\pi_{i}(W)=0$ for $1<i<k$ and $H_{k}(W ; \mathbb{Z})=0$ but $\pi_{k}(W) \neq 0$. Since we are working in the topological category, we can use an infinite construction to accomplish this. We will construct $W$ as an ascending union $W_{1} \subset W_{2} \subset W_{3} \subset \cdots$ of compact PL submanifolds of $S^{n}$. Each $W_{m}$ will be a regular neighborhood of a copy of $S^{k} \vee S^{1}$. ( $S^{k} \vee S^{1}$ denotes the wedge, or one point union, of $S^{k}$ and $S^{1}$.)

Choose a standard unknotted copy $A_{2} \vee B_{2}$ of $S^{k} \vee S^{1}$ in $S^{n}$, and let $W_{2}$ be a regular neighborhood of $A_{2} \vee B_{2}$. Notice that $\pi_{1}\left(W_{2}\right)$ is an infinite cyclic group; we denote it by $\mathbb{J}$ and use $t$ to denote a generator of $\mathbb{J}$. The groups $\pi_{i}\left(W_{2}\right)$ are all trivial for $1<i<k$, and $\pi_{k}\left(W_{2}\right)$ is naturally isomorphic to $\mathbb{Z}[\mathbb{J}]$, the integral group ring. Inside $W_{2}$ we embed a second copy, $A_{1}$, of $S^{k}$. Choose the embedding in such a way that $A_{1}$ consists of two disjoint parallel copies of $A_{2}$ connected by a tube that goes around $B_{2}$. (Here we need $k<n-1$.) The connection should be made in such a way that $A_{1}$ represents the element $t-1$ in $\pi_{k}\left(W_{2}\right) \cong \mathbb{Z}[\mathbb{J}]$. We also make sure that $A_{1}$ is unknotted in $S^{n}$. Let $B_{1}$ be a circle in $W_{2}$ such that $B_{1}$ is parallel to $B_{2}$ and $A_{1} \vee B_{1}$ is unknotted in $S^{n}$; in fact, let us just say that $B_{1}=B_{2}$. Define $W_{1}$ to be a thin regular neighborhood of $A_{1} \vee B_{1}$ in int $W_{2}$.

Having defined $W_{1} \subset$ int $W_{2}$, we proceed to define $W$. Both $A_{1} \vee B_{1}$ and $A_{2} \vee B_{2}$ are unknotted in $S^{n}$, so there is an isotopy $h_{t}: S^{n} \rightarrow S^{n}, 0 \leq t \leq 1$, with $h_{0}=\mathrm{id}$, and $h_{1}\left(W_{1}\right)=W_{2}$. We recursively define $W_{m}, m \geq 3$, by $W_{m}=h_{1}\left(W_{m-1}\right)$ and define $W=\bigcup_{m=1}^{\infty} W_{m}$. It is obvious that $\pi_{1}(W)=\mathbb{J}$ and $\pi_{i}(W)=0$ for $1<i<k$. In addition, the inclusion $W_{1} \hookrightarrow W_{2}$ induces the trivial homomorphism on $H_{k}$, so $H_{k}(W ; \mathbb{Z})=0$. We claim that the generator of $\pi_{k}\left(W_{1}\right)$ represents a nonzero element of $\pi_{k}(W)$. If not, this generator would be null-homotopic in $W_{m}$ for some $m$. But the generator of $\pi_{k}\left(W_{1}\right)$ goes to $(t-1)^{m-1}$ times the generator of $\pi_{k}\left(W_{m}\right) \cong \mathbb{Z}[\mathbb{J}]$ and thus does not represent the zero element in that group. We have, therefore, constructed an open subset of $S^{n}$ that has the properties needed for our example; it remains only to show that we can arrange things so that $W$ is the complement of a topological knot.

Define $V_{m}=\overline{S^{n}-W_{m}}$. Then $S^{n}-W=\bigcap_{m=1}^{\infty} V_{m}$ and $V_{1} \supset V_{2} \supset V_{3} \supset \cdots$. Now $W_{m}$ collapses to $A_{m} \vee B_{m}$ and $A_{m} \vee B_{m}$ is an unknotted copy of $S^{k} \vee S^{1}$, so $V_{m}$ has a spine $C_{m} \vee D_{m}$ with $C_{m} \cong S^{n-k-1}$ and $D_{m} \cong S^{n-2}$. Figure 1 shows the relationships between $A_{2}, B_{2}, C_{2}$, and $D_{2}$. 


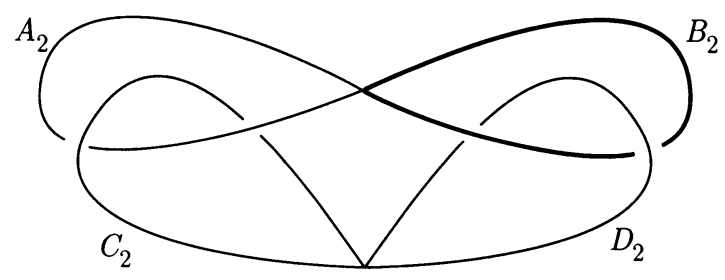

FIGURE 1

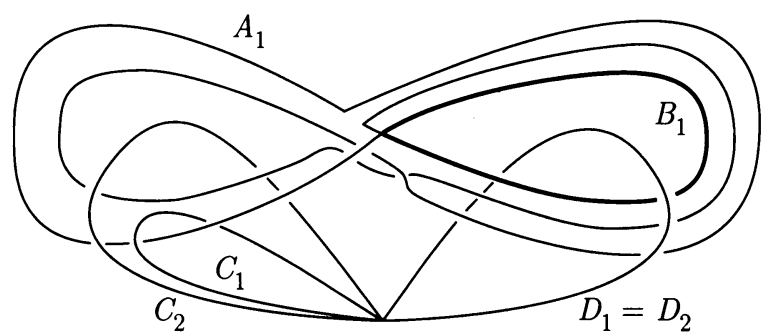

FIGURE 2

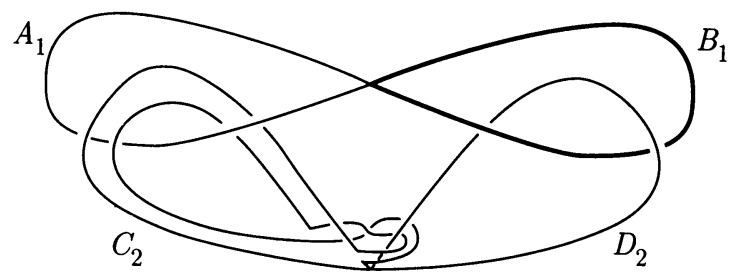

FIGURE 3

Since $B_{m}=B_{m+1}$, we may assume that $D_{m}=D_{m+1}$. We need to understand how $C_{m+1} \vee D_{m+1}$ is embedded in $V_{m}$. We concentrate on the case $m=1$. Figure 2 shows the way in which $C_{2}$ and $D_{2}$ are situated in $V_{1}$ (which is the complement of a regular neighborhood of $A_{1} \vee B_{1}$ ).

In Figure 3 we see what $C_{2} \vee D_{2}$ looks like after we have performed an isotopy that straightens out $A_{1} \vee B_{1}$. As one would expect, $D_{2}=D_{1}$ while $C_{2}$ consists of two copies of $C_{1}$ joined by a tube that winds around $D_{2}$. Since the tube can be made very small, we can arrange things so that $C_{2}$ lies in a close regular neighborhood of $C_{1}$.

We perform an isotopy in each of the regular neighborhoods $V_{m}$ that makes $C_{m+1}$ lie in a close regular neighborhood of $C_{m}$. Then

$$
S^{n}-W=\bigcap_{m=1}^{\infty} V_{m}=C \vee D
$$

where $D=D_{1}$ is a locally flat PL $(n-2)$-sphere and $C=\bigcap_{m=1}^{\infty} N\left(C_{m}\right)$ where each $N\left(C_{m}\right)$ is a regular neighborhood of $C_{m}$. Furthermore, each $C_{m+1}$ is a codimension-3 subpolyhedron of $S^{n}$ and $C_{m+1}$ is null homotopic in $N\left(C_{m}\right)$. It follows that $C$ is a cellular set (by [11] in case $n \neq 4$ or [2] in case $n=4$ ), so we can shrink it to a point; i.e., there is a map $f: S^{n} \rightarrow S^{n}$ whose only nondegenerate point inverse is $C$. The topological knot we are looking for is just $f(D)$. 
Remark 2.1. Let $\epsilon$ denote the end of $W$, and suppose $k \geq \frac{n}{2}$. Since $S^{n}-W$ consists of $C \vee D$ where $D$ is a locally flat PL $(n-2)$-sphere and $C$ is a cellular set of dimension $n-k-1$, it follows that $\pi_{1}(\epsilon) \cong \mathbb{Z}$ and $\pi_{i}(\epsilon)=0$ for $1<i<n-k-1$, but $\pi_{n-k-1}(\epsilon)$ is very complicated. Notice that this fits perfectly with Theorem 1.2 ; because $\pi_{n-k-1}(\epsilon) \neq 0$, it is possible to have $H_{k}(\widetilde{W} ; F) \not H^{n-k-1}(\widetilde{W} ; F)=0$.

Remark 2.2. It is not possible for the first nonstandard homotopy group of a topological knot complement to appear in any dimension above $n-2$. In fact, if we take $k=0$ in Corollary 1.3 we see that $H_{n-1}(\widetilde{W} ; \mathbb{Z})$ is trivial for every knot complement $W$.

\section{DUALITY ON NONCOMPACT MANIFOLDS}

In this section we prove Theorem 1.7. We begin by reviewing the definition of an end of a manifold. An end of a noncompact manifold $W$ is a function $\epsilon$ which assigns to each compact subset $X \subset W$ precisely one component $\epsilon(X)$ of the complement $W-X$, subject to the requirement that $\epsilon(X) \supset \epsilon(Y)$ whenever $X \subset Y$. The open set $\epsilon(X)$ is called a neighborhood of the end. The statement that $W$ has one end means that there exist arbitrarily large compact subsets of $W$ which have connected complements.

We now fix some notation that will be assumed for the remainder of this paper. We assume that $W$ is a connected piecewise linear (PL) $n$-manifold with one end $\epsilon$. We assume also that $\lambda$ is a homomorphism from $\pi_{1}(W)$ to an infinite cyclic group $\mathbb{J}$. We use $t$ to denote a fixed generator of $\mathbb{J}$ and write elements of $\mathbb{J}$ multiplicatively; thus $\mathbb{J}=\left\{t^{i} \mid i=0, \pm 1, \pm 2, \ldots\right\}$. Let $p: \widetilde{W} \rightarrow W$ denote the infinite cyclic cover determined by $\lambda$. We will also use the same symbol $t$ to denote the generator of the group of deck transformations of $\widetilde{W}$ which corresponds to $t$. If $U$ is any subset of $W$, we use $U^{*}$ to denote $p^{-1}(U) \subset \widetilde{W}$. Let $F$ be a field, and let $\Lambda$ denote the group algebra $F[\mathbb{J}]$. Notice that the homology group $H_{k}\left(\widetilde{W}, U^{*} ; F\right)$ can be thought of either as a vector space over $F$ or as a module over $\Lambda$. It is important to distinguish between "finitely generated over $F$ " and "finitely generated over $\Lambda$ ".

Choose a sequence $U_{1} \supset U_{2} \supset U_{3} \supset \cdots$ of connected neighborhoods of $\epsilon$ such that $\bigcap_{i=1}^{\infty} U_{i}=\varnothing$. We may assume that the map $p$ is PL and that each $W_{m}=\overline{W-U_{m}}$ is a compact PL manifold with boundary. We can also make $W_{m}$ connected by removing regular neighborhoods of arcs from $U_{m}$. We next build a connected polyhedron $K \subset \widetilde{W}$ such that $K-U_{m}^{*}$ is a finite polyhedron for every $m$ and $p(K)=W$. For each simplex $\sigma$ in $W_{1}$, we select one simplex $\tilde{\sigma}$ in $W_{1}^{*}$ such that $p(\tilde{\sigma})=\sigma$. Connect all the simplices $\tilde{\sigma}$ by a finite number of arcs in $\widetilde{W}$ to form $K_{1}$. Then for each simplex $\tau$ in $W_{2}-W_{1}$, select a simplex $\tilde{\tau}$ that lies above it. It is possible to connect $\tilde{\tau}$ by an arc $\alpha \subset \widetilde{W}-$ int $W_{1}^{*}$ to a simplex in $W_{1}^{*}$. We shift $\alpha \cup \tilde{\tau}$ by $t^{i}$ for some $i$ so that $(\alpha \cup \tilde{\tau}) \cap K_{1} \neq \varnothing$ and add $\alpha \cup \tilde{\tau}$ to $K_{1}$ to form $K_{2}$. Continuing in the same way, for each $m$ we construct a finite connected polyhedron $K_{m} \subset \widetilde{W}$ such that $p\left(K_{m}\right)=W_{m}$ and $p\left(K_{m}-K_{m-1}\right) \subset W_{m}$-int $W_{m-1}$. Define $K=\bigcup K_{m}$. Notice that, because $K-U_{m}^{*}$ is a finite polyhedron, there exists a positive integer $c(m)$ such that $\left(K-U_{m}^{*}\right) \cap t^{i}\left(K-U_{m}^{*}\right)=\varnothing$ whenever $|i| \geq c(m)$. For each pair of integers 
$(q, m), m \geq 1$, define

$$
\begin{aligned}
N_{q} & =\bigcup_{i \geq q} t^{i}(K), \\
N_{q}^{\prime} & =\bigcup_{i \leq-q} t^{i}(K), \\
E_{q, m} & =N_{q} \cup U_{m}^{*} \cup N_{q}^{\prime} .
\end{aligned}
$$

Then $\widetilde{W}-E_{q, m}$ has compact closure for every pair $(q, m)$ and $\cap E_{q, m}=\varnothing$. Hence

$$
H_{c}^{k}(\widetilde{W} ; F)=\lim _{q, m \rightarrow \infty} H^{k}\left(\widetilde{W}, E_{q, m} ; F\right)
$$

Lemma 3.1. If $H_{i}(\widetilde{W}, \tilde{\epsilon} ; F)$ is profinitely generated over $F$, then the sequence $\left\{U_{m}\right\}$ can be chosen in such a way that the inclusion-induced image of $H_{i}\left(N_{0}, N_{0} \cap U_{m+1}^{*} ; F\right)$ in $H_{i}\left(N_{0}, N_{0} \cap U_{m}^{*} ; F\right)$ is finite dimensional over $F$.

Proof. We use the hypothesis to choose $\left\{U_{m}\right\}$ so that the image of $H_{i}\left(\widetilde{W}, U_{m+1}^{*} ; F\right)$ in $H_{i}\left(\widetilde{W}, U_{m}^{*} ; F\right)$ is finitely generated over $F$ for each $m$. Define $N=N_{0} \cap N_{0}^{\prime}$. Consider the following commutative diagram in which each row is part of an exact Mayer-Vietoris sequence. (Coefficients in $F$ are understood.)

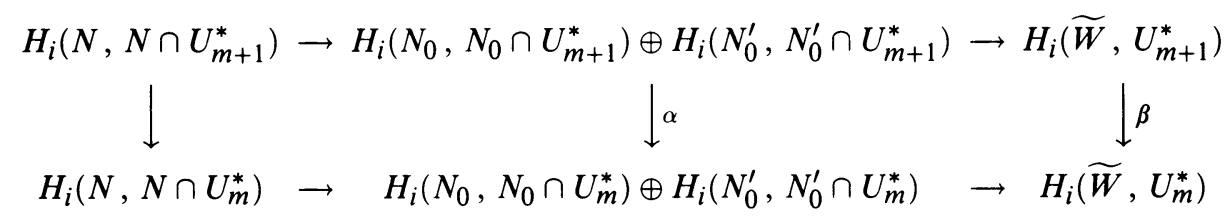

Since $H_{i}\left(N, N \cap U_{m}^{*} ; F\right)$ is finite dimensional (by excision and the choice of $K$ ) and the image of $\beta$ is finite dimensional, it follows that the image of $\alpha$ is finite dimensional.

Lemma 3.2. If $H_{i}(\widetilde{W}, \tilde{\epsilon} ; F)$ and $H_{i-1}(\widetilde{W}, \tilde{\epsilon} ; F)$ are both profinitely generated over $F$, then the sequence $\left\{U_{m}\right\}$ can be chosen in such a way that the inclusion-induced image of $H_{i}\left(\widetilde{W}, N_{0} \cup U_{m+2}^{*} ; F\right)$ in $H_{i}\left(\widetilde{W}, N_{0} \cup U_{m}^{*} ; F\right)$ is finite dimensional over $F$.

Proof. Using the hypothesis and Lemma 3.1 we can choose $\left\{U_{m}\right\}$ in such a way that for each $m$ the inclusion-induced images of $H_{i}\left(\widetilde{W}, U_{m+1}^{*} ; F\right)$ in $H_{i}\left(\widetilde{W}, U_{m}^{*} ; F\right)$ and $H_{i-1}\left(N_{0}, N_{0} \cap U_{m+1}^{*} ; F\right)$ in $H_{i-1}\left(N_{0}, N_{0} \cap U_{m}^{*} ; F\right)$ are finitely generated over $F$. Consider the following diagram in which each row is part of the long exact sequence of a triple.

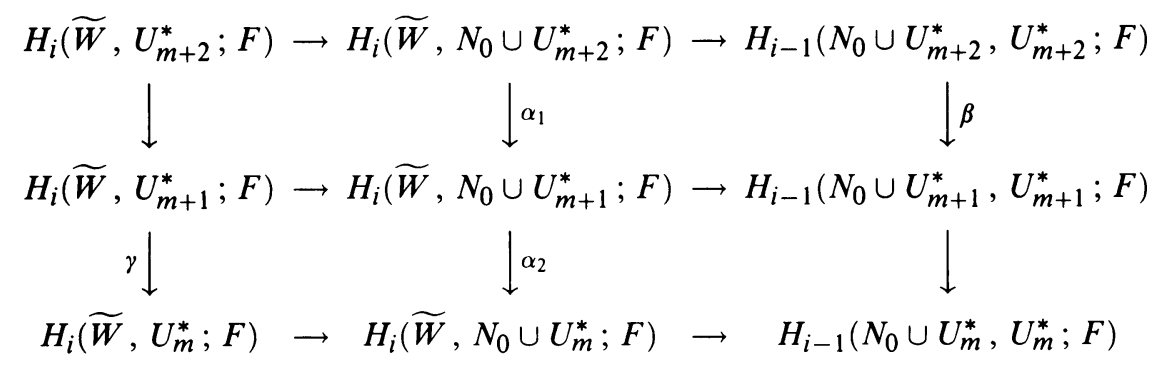


Since both $\beta$ and $\gamma$ have finite-dimensional images, an elementary linear algebra argument shows that $\alpha_{2} \circ \alpha_{1}$ has finite-dimensional image as well.

Lemma 3.3. If $H_{i}(\widetilde{W}, \tilde{\epsilon} ; F)$ and $H_{i-1}(\widetilde{W}, \tilde{\epsilon} ; F)$ are both profinitely generated over $F$, then for each $m$ there is a positive integer $s$ such that the inclusioninduced homomorphism $H_{i}\left(\widetilde{W}, N_{s} \cup U_{m+2}^{*} ; F\right) \rightarrow H_{i}\left(\widetilde{W}, N_{0} \cup U_{m}^{*} ; F\right)$ is trivial. Proof. By Lemma 3.2, the image of $H_{i}\left(\widetilde{W}, N_{0} \cup U_{m+2}^{*} ; F\right)$ in the group $H_{i}\left(\widetilde{W}, N_{0} \cup U_{m}^{*} ; F\right)$ is finitely generated. We choose $s$ large enough so that $N_{-s}$ contains a representative of each of these generators. Then the homomorphism $H_{i}\left(\widetilde{W}, N_{0} \cup U_{m+2}^{*} ; F\right) \rightarrow H_{i}\left(\widetilde{W}, N_{-s} \cup U_{m}^{*} ; F\right)$ is trivial. We shift everything by $t^{s}$ in order to reach the desired conclusion.

Lemma 3.4. If $H_{i}(\widetilde{W}, \tilde{\epsilon} ; F)$ and $H_{i-1}(\widetilde{W}, \tilde{\epsilon} ; F)$ are both profinitely generated over $F$, then $\lim _{m, q \rightarrow \infty} H^{i}\left(\widetilde{W}, N_{q} \cup U_{m}^{*} ; F\right)=0$.

Proof. By Lemma 3.3, $H_{i}\left(\widetilde{W}, N_{s} \cup U_{m+2}^{*} ; F\right) \rightarrow H_{i}\left(\widetilde{W}, N_{0} \cup U_{m}^{*} ; F\right)$ is trivial. Shifting by $t^{q}$ gives $H_{i}\left(\widetilde{W}, N_{q+s} \cup U_{m+2}^{*} ; F\right) \rightarrow H_{i}\left(\widetilde{W}, N_{q} \cup U_{m}^{*} ; F\right)$ trivial for every $q$. Since we are using coefficients in a field, it follows that

$$
H^{i}\left(\widetilde{W}, N_{q} \cup U_{m}^{*} ; F\right) \rightarrow H^{i}\left(\widetilde{W}, N_{q+s} \cup U_{m+2}^{*} ; F\right)
$$

is trivial. (This is one of the points in the proof at which it is convenient to be working over a field.)

Proof of Theorem 1.7. By Poincaré Duality,

$$
\begin{aligned}
H_{k}(\widetilde{W} ; F) & \cong H_{c}^{n-k}(\widetilde{W} ; F) \\
& =\lim _{q, m \rightarrow \infty} H^{n-k}\left(\widetilde{W}, E_{q, m} ; F\right) .
\end{aligned}
$$

Consider the exact Mayer-Vietoris sequence

$$
\begin{aligned}
\cdots & \rightarrow H^{n-k-1}\left(\widetilde{W}, N_{q} \cup U_{m}^{*} ; F\right) \oplus H^{n-k-1}\left(\widetilde{W}, N_{q}^{\prime} \cup U_{m}^{*} ; F\right) \\
& \rightarrow H^{n-k-1}\left(\widetilde{W}, U_{m}^{*} ; F\right) \rightarrow H^{n-k}\left(\widetilde{W}, E_{q, m} ; F\right) \\
& \rightarrow H^{n-k}\left(\widetilde{W}, N_{q} \cup U_{m}^{*} ; F\right) \oplus H^{n-k}\left(\widetilde{W}, N_{q}^{\prime} \cup U_{m}^{*} ; F\right) \rightarrow \cdots
\end{aligned}
$$

By Lemma 3.4, the direct limits of the first and last terms are trivial. Therefore the middle two terms have isomorphic direct limits.

\section{HOMOTOPY PROPERTIES OF KNOT COMPLEMENTS}

In this section we prove Theorems 1.2 and 1.5. We continue to use the notation of $\S 3$, but we need some additional definitions. If $\epsilon$ is an end of $W$, then $\pi_{i}(\epsilon)$ is defined to be the inverse system of groups $\left\{\pi_{i}(U)\right\}$, where $U$ ranges over all neighborhoods of $\epsilon$. Let $G$ be a group. The statement $\pi_{i}(\epsilon) \cong G$ means that the system $\left\{\pi_{i}(U)\right\}$ is stable and its inverse limit is isomorphic to $G$. If $W$ is a topological knot complement, the Hurewicz homomorphism from $\pi_{1}(W)$ to $H_{1}(W ; \mathbb{Z})$ provides a natural homomorphism $\lambda: \pi_{1}(W) \rightarrow$ $\mathbb{J}$. Whenever a covering space of a knot complement is mentioned, it will be 
assumed that the cover is the infinite cyclic cover associated with the Hurewicz homomorphism.

For the remainder of this section we will assume that $h: S^{n-2} \rightarrow S^{n}$ is a topological embedding, that $\Sigma$ denotes $h\left(S^{n-2}\right)$, that $W=S^{n}-\Sigma$, and that $\epsilon$ denotes the end of $W$.

Lemma 4.1. If $i<n-2$ and $\pi_{j}(\epsilon) \cong \pi_{j}\left(S^{1}\right)$ for $j \leq i$, then $H_{i+1}(\widetilde{W}, \tilde{\epsilon} ; F)$ is profinitely generated over $F$ for every field $F$.

Proof. We first choose a sequence of neighbohoods $\left\{U_{m}\right\}$ of $\epsilon$ so that the following conditions are satisfied for each $m \geq 1$.

(1) The closure $\bar{U}_{m}$ of $U_{m}$ in $S^{n}$ is a compact PL manifold neighborhood of $\Sigma$. (Notice that $\bar{U}_{m}=\partial U_{m} \cup U_{m} \cup \Sigma$.)

(2) $\bar{U}_{m+1} \subset \operatorname{int}\left(\bar{U}_{m}\right)$.

(3) The inclusion map $\bar{U}_{m+1} \hookrightarrow \operatorname{int}\left(\bar{U}_{m}\right)$ factors up to homotopy through a retraction of $\bar{U}_{m+1}$ to $\Sigma$.

(4) $\cap U_{m}=\varnothing$.

Using the hypothesis $\pi_{j}(\epsilon) \cong \pi_{j}\left(S^{1}\right)$ for $j \leq i$ we can further choose $\left\{U_{m}\right\}$ so that the image of $\pi_{j}\left(U_{m+1}\right)$ in $\pi_{j}\left(U_{m}\right)$ is trivial in case $1<j \leq i$ or $\mathbb{J}$ in case $j=1$. It follows that

(5) the image of $H_{i}\left(U_{m+1}^{*} ; F\right)$ in $H_{i}\left(U_{m}^{*} ; F\right)$ is trivial.

By excision we have $H_{i+1}\left(W, U_{m} ; F\right) \cong H_{i+1}\left(S^{n}, \bar{U}_{m} ; F\right)$. The diagram

$$
\begin{aligned}
H_{i+1}\left(S^{n}, \bar{U}_{m+1} ; F\right) & \longrightarrow \widetilde{H}_{i}\left(\bar{U}_{m+1} ; F\right) \\
\downarrow & \\
0=H_{i+1}\left(S^{n}\right) \longrightarrow H_{i+1}\left(S^{n}, \bar{U}_{m} ; F\right) & \longrightarrow \widetilde{H}_{i}\left(\bar{U}_{m} ; F\right)
\end{aligned}
$$

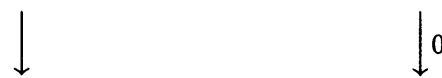

shows that the inclusion-induced homomorphism $H_{i+1}\left(S^{n}, \bar{U}_{m+1} ; F\right) \rightarrow$ $H_{i+1}\left(S^{n}, \bar{U}_{m} ; F\right)$ is trivial. Hence $H_{i+1}\left(W, U_{m+1} ; F\right) \rightarrow H_{i+1}\left(W, U_{m} ; F\right)$ is trivial as well.

Let

$$
\alpha_{m}: H_{i+1}\left(\widetilde{W}, U_{m+1}^{*} ; F\right) \rightarrow H_{i+1}\left(\widetilde{W}, U_{m}^{*} ; F\right)
$$

and

$$
\beta_{m}: H_{i+1}(\widetilde{W} ; F) \rightarrow H_{i+1}\left(\widetilde{W}, U_{m}^{*} ; F\right)
$$

denote the inclusion-induced homomorphisms. Then the diagram

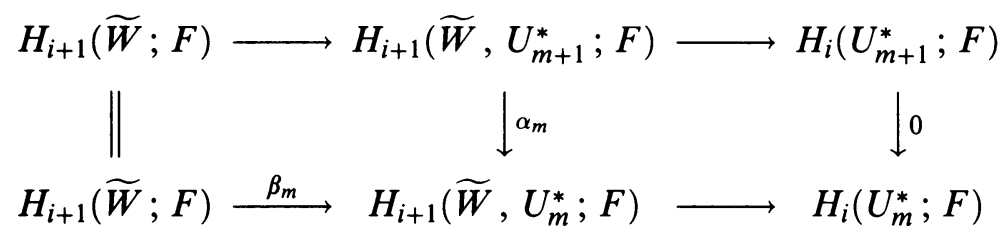

shows that $\operatorname{im}\left(\alpha_{m}\right)=\operatorname{im}\left(\beta_{m}\right)$. Now $U_{m+2}$ is related to $U_{m}$ in exactly the same way that $U_{m+1}$ is, so we also have that $\operatorname{im}\left(\alpha_{m} \circ \alpha_{m+1}\right)=\operatorname{im}\left(\beta_{m}\right)=\operatorname{im}\left(\alpha_{m}\right)$. It follows that $\operatorname{im}\left(\alpha_{m}\right)=\operatorname{im}\left(\alpha_{m} \circ \alpha_{m+1} \circ \cdots \circ \alpha_{m+j}\right)$ for every $j>0$.

The exact sequence

$$
0 \rightarrow C_{*}\left(\widetilde{W}, U_{m}^{*} ; F\right) \stackrel{t-1}{\longrightarrow} C_{*}\left(\widetilde{W}, U_{m}^{*} ; F\right) \stackrel{p_{*}}{\longrightarrow} C_{*}\left(W, U_{m} ; F\right) \rightarrow 0
$$


of chain complexes gives rise to a long exact sequence

$$
\cdots \rightarrow H_{i+1}\left(\widetilde{W}, U_{m}^{*} ; F\right) \stackrel{t-1}{\longrightarrow} H_{i+1}\left(\widetilde{W}, U_{m}^{*} ; F\right) \stackrel{p_{*}}{\longrightarrow} H_{i+1}\left(W, U_{m} ; F\right) \rightarrow \cdots
$$

of homology groups. Therefore we have the following commutative diagram with exact rows:

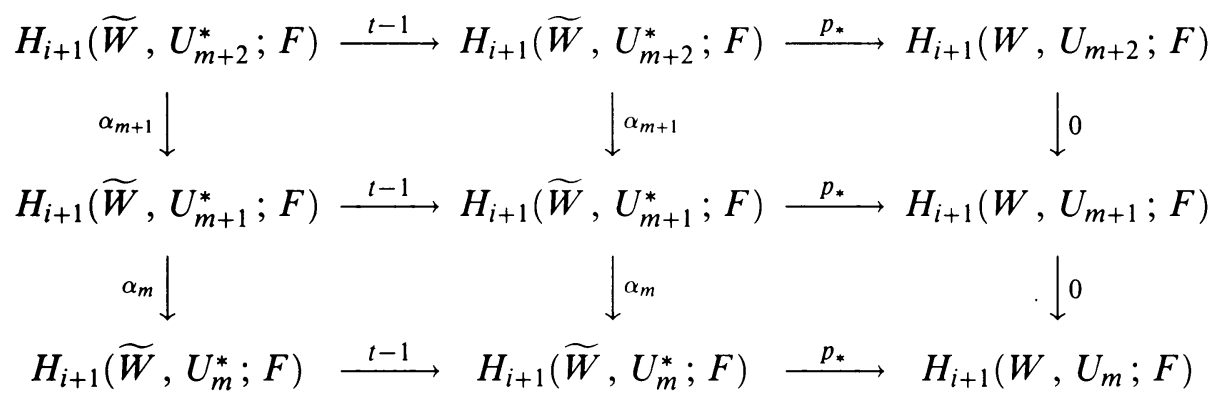

The upper half of the diagram shows that

$$
\operatorname{im}\left(\alpha_{m+1}\right) \subset(t-1)\left(H_{i+1}\left(\widetilde{W}, U_{m+1}^{*} ; F\right)\right)=\operatorname{im}(t-1) .
$$

Thus $\operatorname{im}\left(\alpha_{m}\right)=\operatorname{im}\left(\alpha_{m} \circ \alpha_{m+1}\right) \subset \operatorname{im}\left(\alpha_{m} \circ(t-1)\right)=(t-1) \operatorname{im}\left(\alpha_{m}\right)$. It follows that $\operatorname{im}\left(\alpha_{m}\right)=(t-1) \operatorname{im}\left(\alpha_{m}\right)$. Now $\operatorname{im}\left(\alpha_{m}\right)$ is a subspace of $H_{i+1}\left(\widetilde{W}, U_{m+1}^{*} ; F\right)$ which is finitely generated over $\Lambda$. Since $\operatorname{im}\left(\alpha_{m}\right)$ is finitely generated over $\Lambda$ and $\operatorname{im}\left(\alpha_{m}\right)=(t-1) \operatorname{im}\left(\alpha_{m}\right)$, it follows that $\operatorname{im}\left(\alpha_{m}\right)$ is finite dimensional over $F$. (See the proof of Assertion 5, p. 118, of [12]. This is one point in the proof at which it is essential to be working over a field.) Hence $H_{i+1}(\widetilde{W}, \tilde{\epsilon} ; F)$ is profinitely generated over $F$.

Proof of Theorem 1.2. Let $\left\{U_{m}\right\}$ be as in the proof of Lemma 4.1. We claim that $\lim _{\longrightarrow} \widetilde{H}^{i}\left(U_{m}^{*} ; F\right)$ is trivial whenever $i \leq n-k-1$. Each $U_{m}$ contains a representative of the generator of $H_{1}(W)$, so each $U_{m}^{*}$ is connected (see [9, Lemma 3.6]) and therefore $\lim \widetilde{H}^{0}\left(U_{m}^{*} ; F\right)=0$. If $\pi_{1}(\epsilon) \cong \mathbb{Z}$, then $\pi_{1}(\epsilon)$ is canonically isomorphic to $\overrightarrow{H_{1}(W)}$ (again, see [9, Lemma 3.6]). It follows that the inclusion-induced homomorphism $\pi_{1}\left(U_{m+1}^{*}\right) \rightarrow \pi_{1}\left(U_{m}^{*}\right)$ is trivial whenever $\pi_{1}(\epsilon) \cong \mathbb{Z}$ and so $\lim _{\longrightarrow} \widetilde{H}^{1}\left(U_{m}^{*} ; F\right)=0$. Since $\pi_{i}(\epsilon)=0$ for $2 \leq i \leq n-k-1$, the claim is now clear.

From Lemma 4.1 and Theorem 1.7 we see that

$$
H_{k}(\widetilde{W} ; F) \cong H^{n-k-1}(\widetilde{W}, \tilde{\epsilon} ; F) .
$$

Consider the following portion of the exact sequence of the pair $\left(\widetilde{W}, U_{m}^{*}\right)$.

$$
\begin{aligned}
H^{n-k-2}\left(U_{m}^{*} ; F\right) & \rightarrow H^{n-k-1}\left(\widetilde{W}, U_{m}^{*} ; F\right) \\
& \longrightarrow \widetilde{H}^{n-k-1}(\widetilde{W} ; F) \rightarrow \widetilde{H}^{n-k-1}\left(U_{m}^{*} ; F\right)
\end{aligned}
$$

We take the direct limit as $m \rightarrow \infty$. The claim above implies that the limits of the first and last terms are 0 and so the middle two terms have isomorphic limits.

In the next proof it is convenient to use homology of $\widetilde{W}$ based on infinite (but locally finite) chains. These groups are denoted by $H_{j}^{\infty}(\widetilde{W} ; G)$. For each 
coefficient group $G$ there is a Poincaré Duality isomorphism $H_{j}^{\infty}(\widetilde{W} ; G) \cong$ $H^{n-j}(\widetilde{W} ; G)$.

Proof of Corollary 1.3. Suppose $i \geq n-k-1$. Then $\pi_{j}(\widetilde{W})=0$ for $j \leq i$ and $\pi_{j}(\epsilon)=0$ for $j \leq i-1$, so $H_{n-i-1}^{\infty}(\widetilde{W} ; G)=0$ for every group $G$. Hence it follows from Poincare Duality that $H^{i+1}(\widetilde{W} ; G)=0$ for every group $G$ and so $H_{i}(\widetilde{W} ; \mathbb{Z})$ is torsion free. On the other hand, $H_{i}(\widetilde{W} ; \mathbb{Z}) \otimes_{\mathbb{Z}} \mathbb{Q} \cong H_{i}(\widetilde{W} ; \mathbb{Q})$ is trivial by Theorem 1.2. These two facts, together with a homological algebra argument, show that $H_{i}(\widetilde{W} ; \mathbb{Z})=0$.

Proof of Corollary 1.4. By Corollary 1.3, all the homology of $\widetilde{W}$ vanishes. It follows that $\widetilde{W}$ is contractible, and thus $\pi_{i}(W)=0$ for $i \geq 2$.

Proof of Theorem 1.5. Since $W$ has the homotopy type of $S^{1}$, the infinite cyclic cover and the universal cover are the same. As before, we use $U^{*}$ to denote the preimage in $\widetilde{W}$ of a set $U \subset W$. It is convenient in this proof to use homology with local coefficients. Thus $H_{i}(W, U ; \mathbb{Z}[\mathbb{J}])$ is just different notation for $H_{i}\left(\widetilde{W}, U^{*} ; \mathbb{Z}\right)$. We choose a PL embedded circle $C \subset W$ such that $C \hookrightarrow W$ is a homotopy equivalence.

We begin the proof by choosing a sequence $\left\{W_{m}\right\}$ of compact PL submanifolds of $W$ whose union is $W$ such that for each $m$ there is a strong deformation retraction of $W_{m}$ to $C$ in the interior of $W_{m+1}$. Then the inclusion $W_{m}^{*} \hookrightarrow W_{m+1}^{*}$ is homotopically inessential. In fact, $W_{m}^{*} \hookrightarrow W_{m+1}^{*}$ factors up to proper homotopy through a retraction onto $C^{*} \cong \mathbb{R}^{1}$.

Define $U_{m}=W-W_{m}$. In each row of the following diagram, the first arrow is an excision isomorphism and the second is a Poincare Duality isomorphism.

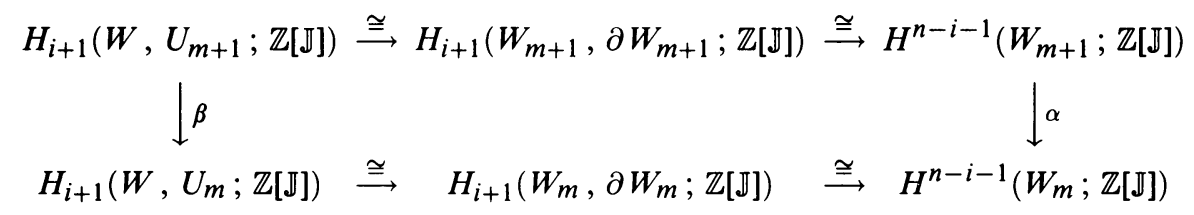

Now $H^{n-i-1}(C ; \mathbb{Z}[\mathbb{J}]) \cong H_{c}^{n-i-1}\left(\mathbb{R}^{1} ; \mathbb{Z}\right)$ and $H_{c}^{n-i-1}\left(\mathbb{R}^{1} ; \mathbb{Z}\right)=0$ for $n-i-$ $1 \neq 1$. It follows that the inclusion-induced homomorphism

$$
\alpha: H^{n-i-1}\left(W_{m+1} ; \mathbb{Z}[\mathbb{J}]\right) \rightarrow H^{n-i-1}\left(W_{m} ; \mathbb{Z}[\mathbb{J}]\right)
$$

is trivial for $n-i-1 \neq 1$ (i.e., for $i \neq n-2)$. Thus $\beta: H_{i+1}\left(W, U_{m+1} ; \mathbb{Z}[\mathbb{J}]\right) \rightarrow$ $H_{i+1}\left(W, U_{m} ; \mathbb{Z}[\mathbb{J}]\right)$ is trivial for $i \neq n-2$. We next look at the diagram below in which each row is part of the long exact sequence of a pair.

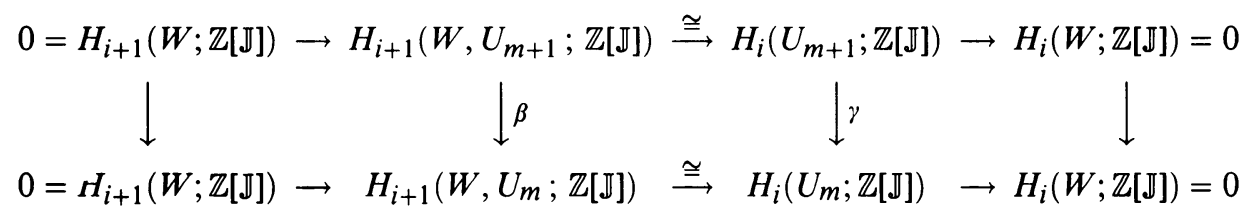

Since $\beta=0$ for $i \neq n-2$, it follows that $\gamma=0$ for $i \neq n-2$. Hence $\gamma: H_{i}\left(U_{m+1} ; \mathbb{Z}[\mathbb{J}]\right) \rightarrow H_{i}\left(U_{m} ; \mathbb{Z}[\mathbb{J}]\right)$ is trivial for every $m$ and for $i \neq n-2$. The Eventual Hurewicz Theorem [1, Proposition 3.1] implies that $\pi_{i}(\epsilon)=0$ 
for $i<n-2$. (This is the point in the proof at which we use the fact that $\pi_{1}(\epsilon) \cong \mathbb{Z}$.)

In case $i=n-2$, we have $H^{n-i-1}(C ; \mathbb{Z}[\mathbb{J}])=H_{c}^{1}\left(\mathbb{R}^{1} ; \mathbb{Z}\right) \cong \mathbb{Z}$. Thus $\operatorname{im}(\alpha) \cong \operatorname{im}(\beta) \cong \operatorname{im}(\gamma) \cong \mathbb{Z}$. It follows that $\pi_{n-2}(\epsilon) \cong \mathbb{Z}$.

Remark. The following special case of Corollary 1.6 should be pointed out explicitly. The corollary has already appeared in both [8] and [9], but it is mentioned again in order to make the point that it is a purely algebraic fact and does not depend on the work of Freedman $[2,3]$ on the topology of 4-manifolds.

Corollary 4.2. Suppose $h: S^{2} \rightarrow S^{4}$ is a topological embedding. Let $W=$ $S^{4}-h\left(S^{2}\right)$, and let $\epsilon$ denote the end of $W$. If $\pi_{1}(W) \cong \mathbb{Z} \cong \pi_{1}(\epsilon)$, then $W$ has the proper homotopy type of $S^{1} \times \mathbb{R}^{3}$.

\section{ACKNOWLEDGMENT}

The author wishes to express his thanks to Vo Thanh Liem for helping to originate many of the ideas in this paper and to the referee for suggesting several improvements.

\section{REFERENCES}

1. S. C. Ferry, Homotoping 6-maps to homeomorphisms, Amer. J. Math. 101 (1979), 567-582.

2. M. H. Freedman, The topology of four-dimensional manifolds, J. Differential Geom. 17 (1982), 357-453.

3. M. H. Freedman and F. Quinn, Topology of 4-manifolds, Princeton Univ. Press, Princeton, NJ, 1990.

4. C. Guilbault, An open collar theorem for 4-manifolds, Trans. Amer. Math. Soc. 331 (1992), 227-245.

5. J. G. Hollingsworth and T. B. Rushing, Homotopy characterization of weakly flat codimension 2 spheres, Amer. J. Math. 98 (1976), 385-394.

6. A. Kawauchi, A partial Poincaré duality theorem for infinite cyclic coverings, Quart. J. Math. Oxford Ser. (2) 26 (1975), 437-458.

7. M. Kervaire and C. Weber, $A$ survey of multidimensional knots, Knot Theory (J. C. Hausmann, ed.), Springer-Verlag, New York and Berlin, 1978, pp. 61-134.

8. V. T. Liem and G. A. Venema, Characterization of knot complements in the 4-sphere, Topology Appl. 42 (1991), 231-245.

9. _ـ On the asphericity of knot complements, Canad. J. Math. 45 (1993), 340-356.

10. J. Levine, Unknotting spheres in codimension two, Topology 4 (1965), 9-16.

11. D. R. McMillan, Jr., A criterion for cellularity in a manifold, Ann. of Math. (2) 79 (1964), 327-337.

12. J. Milnor, Infinite cyclic coverings, Conference on the Topology of Manifolds, Prindle, Weber and Schmidt, Boston, 1968, pp. 115-133.

13. D. W. Sumners, Homotopy torsion in codimension two knots, Proc. Amer. Math. Soc. 24 (1970), 229-240.

Department of Mathematics, Calvin College, Grand Rapids, Michigan 49546

Current address: Department of Mathematics, University of Michigan, Ann Arbor, Michigan 48109-1003

E-mail address: venema@calvin.edu 